\title{
Fuzzy RAM Analysis of the Screening Unit in a Paper Industry by Utilizing Uncertain Data
}

\author{
Harish Garg, Monica Rani, and S. P. Sharma \\ Department of Mathematics, Indian Institute of Technology, Roorkee 247667, Uttarakhand, India \\ Correspondence should be addressed to Harish Garg, harishg58iitr@gmail.com
}

Received 18 July 2012; Accepted 6 October 2012

Academic Editor: Tadashi Dohi

Copyright () 2012 Harish Garg et al. This is an open access article distributed under the Creative Commons Attribution License, which permits unrestricted use, distribution, and reproduction in any medium, provided the original work is properly cited.

Reliability, availability, and maintainability (RAM) analysis has helped to identify the critical and sensitive subsystems in the production systems that have a major effect on system performance. But the collected or available data, reflecting the system failure and repair patterns, are vague, uncertain, and imprecise due to various practical constraints. Under these circumstances it is difficult, if not possible, to analyze the system performance up to desired degree of accuracy. For this, Artificial Bee Colony based Lambda-Tau (ABCBLT) technique has been used for computing the RAM parameters by utilizing uncertain data up to a desired degree of accuracy. Results obtained are compared with the existing Fuzzy Lambda-Tau results and we conclude that proposed results have a less range of uncertainties. Also ranking the subcomponents for improving the performance of the system has been done using RAM-Index. The approach has been illustrated through analyzing the performance of the screening unit of a paper industry.

\section{Introduction}

In any production plant, systems are expected to be operational and available for the maximum possible time so as to maximize the overall production and hence profit. That is each component/system of the entire production plant will run failure free for enhancing the production as well as productivity of the plant and furnish their excellent performance. However, failures are inevitable; a product will fail sooner or later. These failures may be the result of human error, poor maintenance, or inadequate testing and inspection. Therefore, the systems and components undergo several failure-repair cycles that include logistic delays while performing repair leads to the degradation of systems' overall performance [1]. System performance depends on reliability and availability of the system/components, operating environment, maintenance efficiency, operation process and technical expertise of operators, and so forth. To improve the system reliability and availability, implementation of appropriate maintenance strategies play an important role. High performance of these units can be achieved with highly reliable subunits and perfect maintenance. To this effect the knowledge of behavior of system, their component(s) is customary in order to plan and adapt suitable maintenance strategies. Thus, maintainability is also to be a key index to enhance the performance of these systems $[2,3]$. On the other hand availability of the system can be improved by improvement in its reliability and maintainability. To maintain the availability of sophisticated systems to a higher level, the systems structure design or system components of higher availability should be required, or both of them are performed simultaneously. Implementation of these methods to improve the system availability or reliability will normally consume resources such as cost, weight, volume, and so forth. Thus, it is very important for decisionmakers to fully consider both the actual business and the quality requirements. Thus keeping in view the competitive environment, behavior of such systems can be studied in terms of their reliability, availability, and maintainability (RAM).

RAM as an engineering tool evaluates the equipment performance at different stages in design process ad hence play an important role in controlling both the quantity and quality of the products. They aim at estimating and 
predicting the probability of the failure and optimizing the operation management related with the provision of the failures, that is, maintenance policies. Factors that affect RAM of a repairable industrial system include machinery operating conditions, maintenance conditions, infrastructural facilities, and so forth $[2,4]$. The growing complexity of technological systems as well as rapidly increasing operation and maintenance costs incurred due to loss of operation as a consequence of sudden or sporadic failures have brought to the forefront the aspects of RAM associated with the production/manufacturing systems. The expectation today is that complex equipment and systems should not be free from defects and systematic failures but also perform the required function for a stated time interval and should have a fail-safe behavior in case of critical or catastrophic failures. But, failure is nearly an unavoidable phenomenon in mechanical systems/components. For failure analysis variety of methods exists in literature. These include reliability block diagrams (RBDs), Monte Carlo simulation (MCS), Markov Modeling, failure mode and effect analysis, Petri nets, fault tree analysis, and so forth [2, 3, 5-7]. Most of the repairable mechanical systems exhibit constant repair and failure rate after initial burn-in period of bath tub curve. Out of these both FTA and PN recognized as a powerful tool for estimating the reliability of large scaled systems, where system success or failure is described by the state of the top event. The probability of a top event is a function of the failure probability of a primary event, whose data are collected either from the available historical data or raw data provided by the experts. It is often assumed that all probabilities or probability distributions are known or perfectly determinable. This assumption is not consistent with reality because we are faced with different fundamental uncertainties for reliability modeling and analysis of a system in design stage. Thus the data, available from the past record, is incomplete, imprecise, vague, and conflicting; that is, historical records can only represent the past behavior but may be unable to predict future behavior of the equipment, and that leads to inadequate knowledge of basic failure events. Also, the traditional analytical techniques need large amounts of data, which are difficult to obtain because of various practical constraints such as rare events of components, human errors, and economic considerations for the estimation of failure/repair characteristics of the system. In such circumstances, it is usually not easy to analyze the behavior and performance of these systems up to desired degree of accuracy by utilizing available resources, data, and information. These challenges imply that a new and pragmatic approach is needed to access and analyze RAM of these systems because organizational performance and survivability depends a lot on reliability and maintainability of its components/parts and systems.

To this effect, the composite measure of reliability, availability, and maintainability has been introduced, called as RAM-Index, for measuring the system performance by simultaneously considers all the three key indices which influence the system performance directly. Rajpal et al. [8] developed an artificial neural network (ANN) model, by using historical data, for assessing the effect of input parameters on this Index of a repairable system. Their index was static in nature while Komal et al. [9] introduced RAM-Index which was time dependent and used historical uncertain data for its evolution. However, almost all the previous studies were carried out by considering the failure rate of the component which follows the exponential distribution that is, a constant failure rate model. Rani et al. [10] have extended this idea for a time varying failure rate and a constant repair rate model. In the present paper, system performance in terms of RAM-index of a repairable industrial systems has been analyzed by considering the time varying failure rate instead of constant failure rate model. To compute the RAM parameters and consequently RAMIndex by utilizing uncertain, limited, and vague data, an approach gave by Knezevic and Odoom [11] may be used. Based on these the behavior analysis of complex repairable industrial systems are analyzed by the researchers in the form of fuzzy membership functions of various reliability parameters $[12,13]$. In their approach, PN is used to model the system while fuzzy set theory is used to quantify the uncertain, vague, and imprecise data. But it is analyzed from the studies that when this approach is applied for large and complex systems, the computed reliability indices in the form of fuzzy membership function have wide spread (support), that is, high level of uncertainty exists due to various fuzzy arithmetic operations are used in the computations [1417] and hence it does not provide the actual trend of the system behavior. In order to reduce the uncertainty level in the analysis, spread for each reliability index must be reduced up to a desired degree of accuracy so that plant personnel may use these indices to analyze the system behavior more closely and take more sound decisions to improve the performance of the plant. For overcoming this drawback and to generalized the approach for large and complex systems, Artificial Bee Colony based Lambda-Tau (ABCBLT) technique is used in this study [17]. ABCBLT technique is hybridized technique in which Lambda-Tau methodology has been used for obtaining the expression of RAM parameters and Artificial Bee Colony (ABC) [18-20] is used to construct their membership function in the form of triangular fuzzy number by using ordinary arithmetic operation instead of fuzzy arithmetic. Major advantage of the ABCBLT technique is that it give compressed search space for each computed reliability index by utilizing available and uncertain data.

Thus, it is observed from the study that RAM parameters of the system may be calculated by utilizing uncertain, vague, and imprecise data. The objective of the present work is to quantify the uncertainties with the help of fuzzy numbers and to develop an approach for assessing the effect of failure and repair pattern on the composite measure of RAM of industrial systems. For this, a time varying failure rate instead of constant failure rate has been considered for analysis. The approach has been demonstrate through a RAM analysis of the screening unit of a paper industry using $\mathrm{ABC}$ and Lambda-Tau methodology. The computed results are compared with the fuzzy Lambda-Tau results. The sensitivity analysis of the components has been done by using proposed RAM-Index analysis for finding the components as 


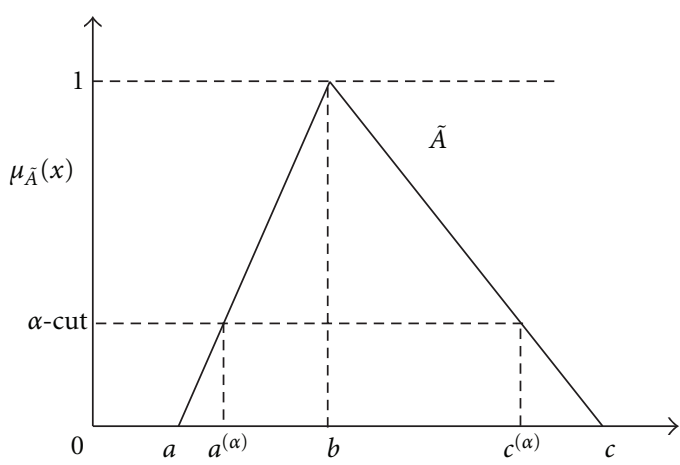

FIgURE 1: Triangular fuzzy number of fuzzy set $\widetilde{A}$.

per the preferential order for improving the production as well as productivity of the system. The rest of the paper is organized as follow: Section 2 describe the basic notations and terms related to fuzzy set theory which will help to analyze the system performance. The generalized RAMIndex has been defined in Section 3 while the ABCBLT technique has been discussed in Section 4. Section 5 discusses the case study of the screening unit of the paper industry for RAM analysis and along with their results. Finally concrete conclusion has been discussed in Section 6 .

\section{Basic Concepts of Fuzzy Set Theory}

Problems in the real world quite often turn out to be complex owing to an element of uncertainty either in the parameters which define the problem or in the situations in which the problem occurs. Although probability theory has been an effective tool to handle uncertainty, it can be applied only to situations whose characteristics are based on random processes, that is, processes in which the occurrence of events is strictly determined by chance. However, in reality, there turn out to be problems, a large class of them whose uncertainty is characterized by a nonrandom process. Here, the uncertainty may arise due to partial information about the problem or due to information which is not fully reliable or due to partial information about the problem. This problem was overcome by using the notion of the fuzzy set introduced by Zadeh in 1965 [21] in the evaluation of the reliability of a system.

2.1. Fuzzy Set. The concept of fuzzy set was introduced by Zadeh [21] in 1965, which can be defined on the universe of discourse $U$ as $\widetilde{A}=\left\{\left\langle x, \mu_{\widetilde{A}}(x)\right\rangle \mid x \in U\right\}$, where $\mu_{\widetilde{A}}$ is the membership function of the fuzzy set $\widetilde{A}$ defined as $\mu_{\tilde{A}}: U \rightarrow$ $[0,1]$ and $\mu_{\tilde{A}}(x)$ indicates the degree of membership of $x$ in $\tilde{A}$ and its value lies between zero and one. When a set is an ordinary set, its membership function can take on only two values 0 and 1 , with $\chi_{A}(x)=1$ or 0 according as $x$ does or does not belong to $A \cdot \chi_{A}(x)$ is referred to as the characteristic function of the set $A$.
TABLE 1: RAM parameters of the system $[10,17]$.

\begin{tabular}{ll}
$\begin{array}{l}\text { Reliability } \\
\text { parameters }\end{array}$ & Expression \\
\hline Reliability & $R_{s}(t)=\exp \left[-\left(\frac{t}{\theta}\right)^{\beta}\right]$ \\
& $A_{s}(t)=\exp \left\{-\left(\frac{t}{\theta}\right)^{\beta}-\frac{t}{\tau}\right\}$ \\
Availability & $\times\left[1+\frac{1}{\tau} \int_{0}^{t} \exp \left\{\left(\frac{t}{\theta}\right)^{\beta}+\frac{t}{\tau}\right\} d t\right]$ \\
& \\
Maintainability & $M_{s}(t)=1-\exp \left(\frac{-t}{\tau}\right)$
\end{tabular}

2.2. Convex Fuzzy Set. A fuzzy set $\tilde{A}$ in universe $U$ is convex if and only if the membership functions $\mu_{\tilde{A}}$ of $\tilde{A}$ is fuzzyconvex, that is,

$$
\begin{aligned}
& \mu_{\widetilde{A}}\left(\lambda x_{1}+(1-\lambda) x_{2}\right) \\
& \quad \geq \min \left(\mu_{\tilde{A}}\left(x_{1}\right), \mu_{\tilde{A}}\left(x_{2}\right)\right), \quad \forall x_{1}, x_{2} \in U, 0 \leq \lambda \leq 1 .
\end{aligned}
$$

2.3. Fuzzy Number. A fuzzy number $\widetilde{A}$ is a convex normalized fuzzy set $\widetilde{A}$ of the real line $\mathbb{R}$ such that

(i) it exists exactly one $x_{0} \in \mathbb{R}$ with $\mu_{\widetilde{A}}\left(x_{0}\right)=1$.

(ii) $\mu_{\tilde{A}}$ is piecewise continuous;

and its membership function is defined as

$$
\mu_{\widetilde{A}}(x)= \begin{cases}f_{A}(x) ; & a \leq x \leq b, \\ 1 ; & x=b, \\ g_{A}(x) ; & b \leq x \leq c, \\ 0 ; & \text { otherwise }\end{cases}
$$

where $0 \leq \mu_{\widetilde{A}}(x) \leq 1$ and $a, b, c \in R$ such that $a \leq b \leq c$, and two functions $f_{A}, g_{A}: R \longrightarrow[0,1]$ are called the sides of fuzzy number. The function $f_{A}$ is nondecreasing continuous functions and the function $g_{A}$ is nonincreasing continuous functions.

2.4. $\alpha$-Cut of the Fuzzy Set. $\alpha$-cut of the fuzzy set $\tilde{A}$, denoted by $A^{(\alpha)}$, is a crisp set which consists of elements of $\tilde{A}$ having at least degree $\alpha$ as is defined mathematically as

$$
A^{(\alpha)}=\left\{x \in U: \mu_{\widetilde{A}}(x) \geq \alpha\right\}
$$

where $\alpha$ is the parameter in the range $0 \leq \alpha \leq 1$. Every $\alpha$-cut of a fuzzy number is a closed interval and a family of such intervals describes completely a fuzzy number under study. Hence we have $A^{(\alpha)}=\left[A_{L}^{(\alpha)}, A_{U}^{(\alpha)}\right]$, where

$$
\begin{aligned}
& A_{L}^{(a)}(x)=\inf \left\{x \in R: \mu_{\tilde{A}}(\mathrm{x}) \geq \alpha\right\}, \\
& A_{U}^{(a)}(x)=\sup \left\{x \in R: \mu_{\tilde{A}}(\mathrm{x}) \geq \alpha\right\} .
\end{aligned}
$$




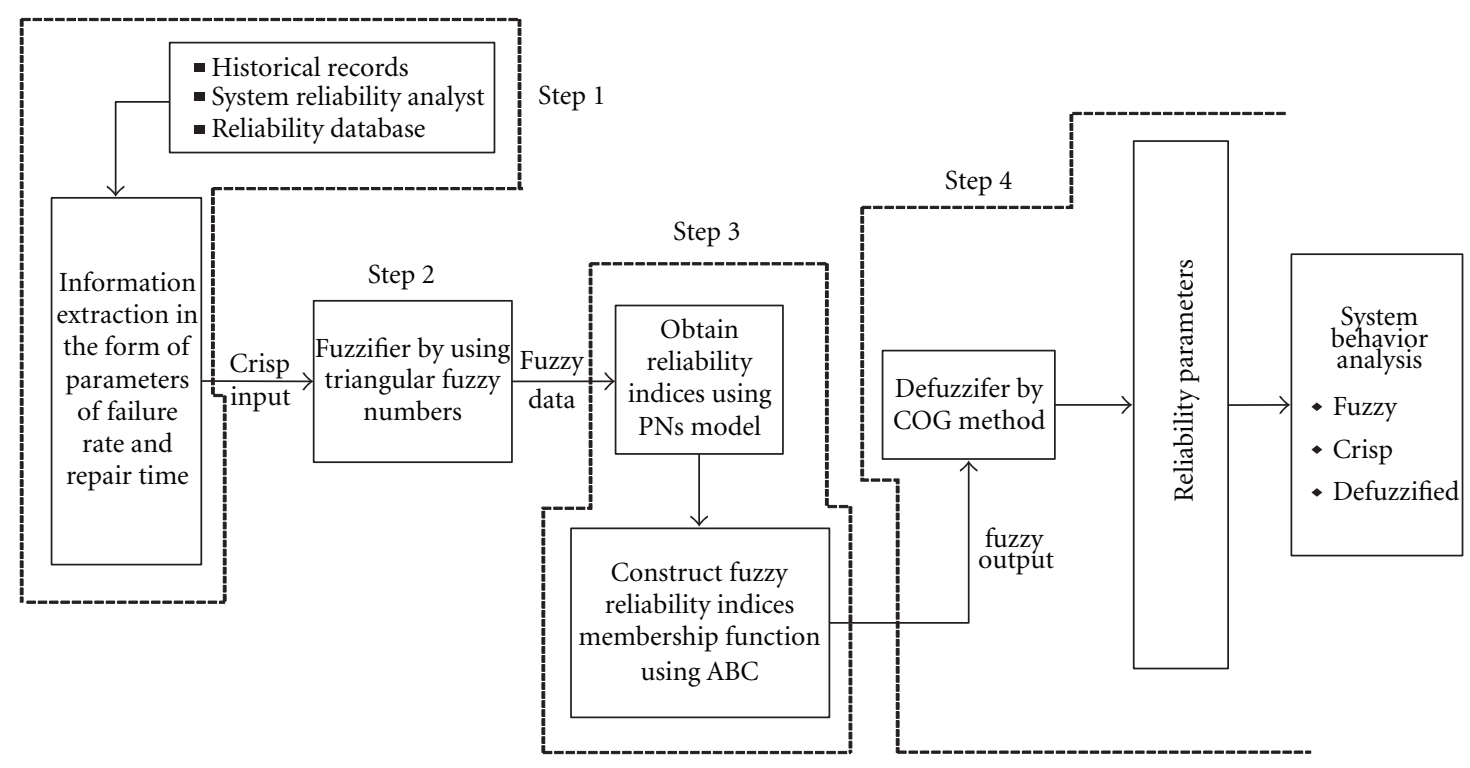

FIgURE 2: Flow chart of the ABCBLT methodology.

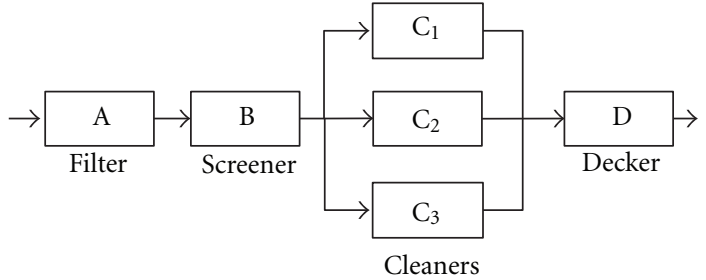

(a)

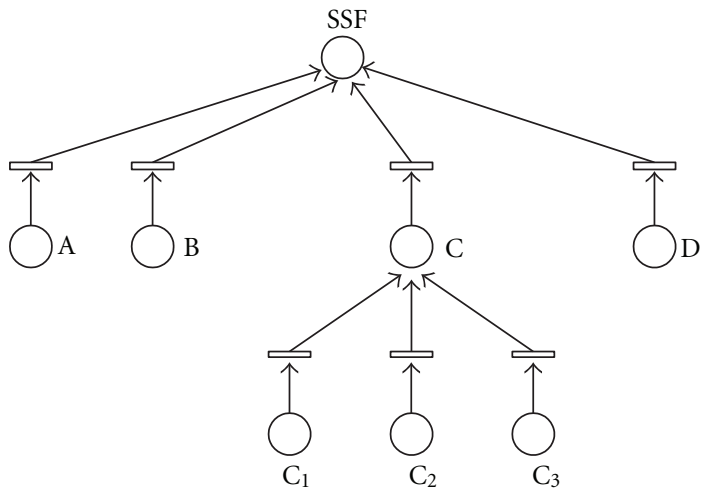

(b)

FIGURE 3: Screening system: (a) RBD and (b) PN model.

2.5. Triangular Fuzzy Number and Interval Arithmetic Operations. The concept of membership function is most important aspect in fuzzy set theory. They are used to represent various fuzzy sets. Many membership functions such as normal, triangular, trapezoidal can be used to represent fuzzy numbers. However, triangular membership functions (TMF) are widely used for calculating and interpreting reliability data because of their simplicity and understandability [22, 23]. The decision of selecting triangular fuzzy numbers (TFNs) lies in their ease to represent the membership function effectively and to incorporate the judgement distribution of multiple experts. This is not true for complex membership functions, such as trapezoidal one, and so forth. For instance, imprecise or incomplete information such as low/high failure rate that is about 4 or between 5 and 7, is well represented by TMF. In the present paper triangular membership function is used as it not only conveys the behavior of system parameters but also reflect the dispersion of the data adequately. A triangular fuzzy number $\tilde{A}$ with parameters $a \leq b \leq c$, denoted as $\tilde{A}=\langle[(a, b, c) ; \mu]\rangle$ in the set of the real number $\mathbb{R}$ and its membership function is given as

$$
\mu_{\widetilde{A}}(x)= \begin{cases}\frac{x-a}{b-a} ; & a \leq x \leq b, \\ 1 ; & x=b, \\ \frac{c-x}{c-b} ; & b \leq x \leq c, \\ 0 ; & \text { otherwise. }\end{cases}
$$

The $\alpha$-cuts of the triangular fuzzy set is defined in the closed interval form as below and shown in graphically in Figure 1:

$$
A_{\alpha}=\left[a^{(\alpha)}, c^{(\alpha)}\right]=[(b-a) \alpha+a,-(c-b) \alpha+c] .
$$

The basic arithmetic operations, that is, addition, subtraction, multiplication and division, of fuzzy numbers depends upon the arithmetic of the interval of confidence. The four main arithmetic operation on two triangular fuzzy 
TABLe 2: Basic expression of the Lambda-Tau methodology.

\begin{tabular}{lccc}
\hline Gate & $\lambda_{\mathrm{AND}}$ & $\tau_{\mathrm{AND}}$ & $\lambda_{\mathrm{OR}}$ \\
\hline Expression & $\prod_{j=1}^{n} \lambda_{j}\left[\sum_{i=1}^{n} \prod_{\substack{i=1 \\
i \neq j}}^{n} \tau_{i}\right]$ & $\frac{\prod_{i=1}^{n} \tau_{i}}{\sum_{j=1}^{n}\left[\prod_{\substack{i=1 \\
i \neq j}}^{n} \tau_{i}\right]}$ & $\sum_{i=1}^{n} \lambda_{i}$ \\
\hline
\end{tabular}

(1) Objective function: $f(\mathbf{x}), \quad \mathbf{x}=\left(x_{1}, x_{2}, \ldots, x_{d}\right)$

(2) Generate an initial bee population (solution) $x_{h}$ where $x_{h}=\left(x_{h 1}, x_{h 2}, \ldots, x_{h d}\right)$ and number of employed bees are equal to onlooker bees

(3) Evaluate fitness value

(4) Initialize cycle $=1$

(5) For each employed bee

(a) Produce new food source position $v_{h j}$ in the neighborhood of $x_{h j}$ by $v_{h j}=x_{h j}+u\left(x_{h j}-x_{k j}\right)$

where $k$ is a solution in the neighborhood of selected parameter $j, u$ is random number in the range $[-1,1]$

(b) Evaluate the fitness value at new source $v_{h j}$

(c) If new position is better than previous position then memorizes the new position

(6) End For

(7) Calculate the probability values $p_{h}=f_{h} / \sum_{h=1}^{N} f_{h}$ for the solution where $N$ is the total number of food sources

(8) For each onlooker bee

(a) Chooses a food source depending on $p_{h}$ for the solutions $x_{h}$

(b) Produce new food source positions $v_{h}$ from the populations $x_{h}$ depending upon $p_{h}$ and evaluate their fitness

(c) If new position better than previous position, then memorizes the new position

(9) End For

(10) If there is any abandoned solution that is, if employed bee becomes scout then replace its position with a new random source positions

(11) Memorize the best solution achieved so far

(12) cycle $=$ cycle +1

(13) If termination criterion is satisfied then stop otherwise go to step 5

Algorithm 1: Pseudo code of the ABC algorithm.

sets $\widetilde{A}$ and $\widetilde{B}$ described by the $\alpha$-cuts are given below for the following intervals:

$$
A^{(\alpha)}=\left[A_{1}^{(\alpha)}, A_{3}^{(\alpha)}\right], \quad B^{(\alpha)}=\left[B_{1}^{(\alpha)}, B_{3}^{(\alpha)}\right], \quad \alpha \in[0,1]
$$

(i) Addition: $\widetilde{A}+\widetilde{B}=\left[A_{1}^{(\alpha)}+B_{1}^{(\alpha)}, A_{3}^{(\alpha)}+B_{3}^{(\alpha)}\right]$.

(ii) Subtraction: $\widetilde{A}-\widetilde{B}=\left[A_{1}^{(\alpha)}-B_{3}^{(\alpha)}, A_{3}^{(\alpha)}-B_{1}^{(\alpha)}\right]$.

(iii) Multiplication: $\widetilde{A} \cdot \widetilde{B}=\left[P^{(\alpha)}, Q^{(\alpha)}\right]$, where $P^{(\alpha)}=\min \left(A_{1}^{(\alpha)} \cdot B_{1}^{(\alpha)}, A_{1}^{(\alpha)} \cdot B_{3}^{(\alpha)}, A_{3}^{(\alpha)} \cdot B_{1}^{(\alpha)}\right.$, $\left.A_{3}^{(\alpha)} \cdot B_{3}^{(\alpha)}\right)$ and $Q^{(\alpha)}=\max \left(A_{1}^{(\alpha)} \cdot B_{1}^{(\alpha)}, A_{1}^{(\alpha)} \cdot B_{3}^{(\alpha)}, A_{3}^{(\alpha)}\right.$. $\left.B_{1}^{(\alpha)}, A_{3}^{(\alpha)} \cdot B_{3}^{(\alpha)}\right)$

(iv) Division: $\widetilde{A} \div \widetilde{B}=\widetilde{A} \cdot 1 / \widetilde{B}$, if $0 \notin \widetilde{B}$.

\section{Generalized RAM-Index}

In order to keep the production and productivity of the system high, it is necessary that the system should operate for long run period without failure. But unfortunately failure is an unavoidable phenomenon in an industrial systems. The failure of subsystem or unit will reduce the efficiency of the system and hence maintainability is essential for it.
So it is necessary for the system analyst that in order to increase the performance of the system, current condition of equipments and subsystems should be changed according to time and the need of effective maintenance program. But the problem to the system analyst is that how to find the component on which more attention should be given to save money, manpower, and time. This problem can be resolved by using the RAM analysis using proposed RAM-Index. The proposed RAM-Index has been valid for a component whose failure rate follows the Weibull distribution while repair time follows the exponential distribution. Major advantage of this index is that by varying the component's failure and repair rate parameters the corresponding effect on the system performance has been observed.

Thus, the generalized RAM-Index for analyzing the performance of the system is given in (8)

$$
\operatorname{RAM}(t)=w_{1} \times R_{s}(t)+w_{2} \times A_{s}(t)+w_{3} \times M_{s}(t),
$$

where $R_{s}, A_{s}$, and $M_{s}$ are, respectively, the reliability, availability, and maintainability of the system whose expressions are given in the Table 1 and $w_{i} \in(0,1), i=1,2,3$ are weights such that $\sum_{i=1}^{3} w_{i}=1$. The value of $w=[0.36,0.30,0.34]$ has been used for calculating RAM-Index which is same as used by the researchers $[8,9]$. 


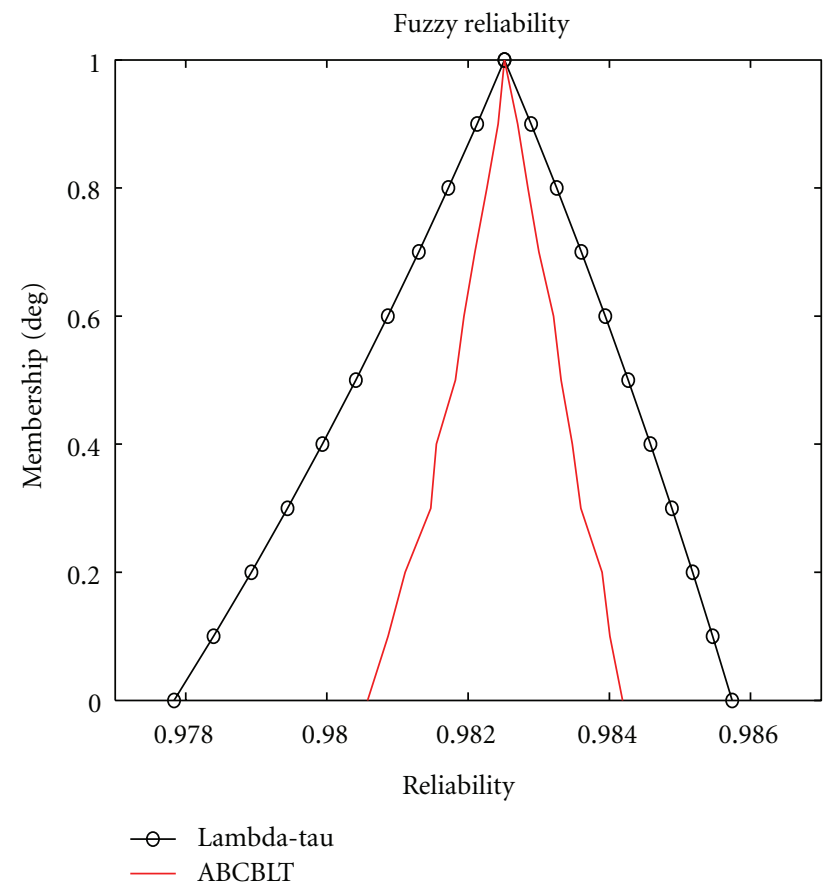

(a)

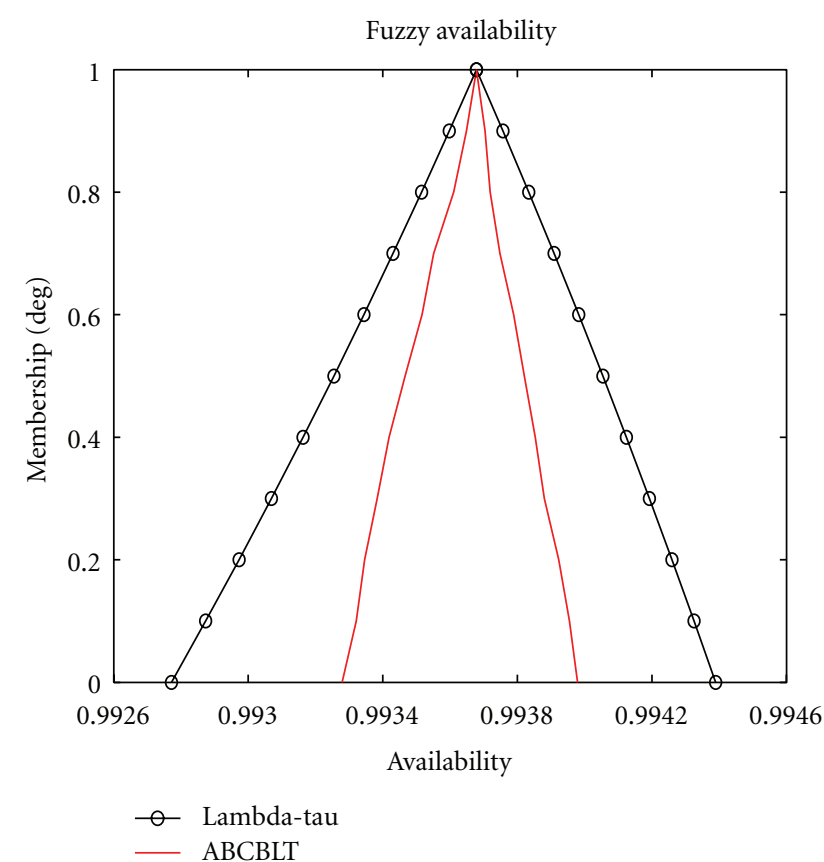

(b)

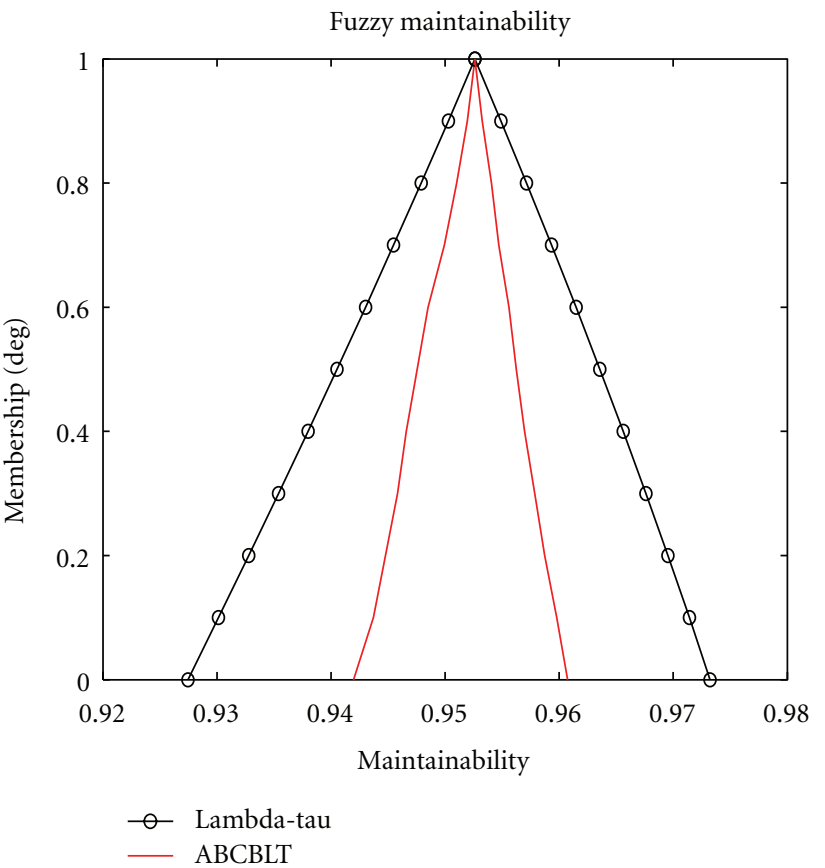

(c)

Figure 4: Fuzzy RAM parameters plot at $\pm 15 \%$ spreads.

Equation (8) can be rewritten in more elaborative form as

$$
\begin{aligned}
\operatorname{RAM}(t)= & w_{1} \times \exp \left[-\left(\frac{t}{\theta}\right)^{\beta}\right]+w_{3} \times 1-\exp \left(\frac{-t}{\tau}\right) \\
& +w_{2} \times \exp \left\{-\left(\frac{t}{\theta}\right)^{\beta}-\frac{t}{\tau}\right\}
\end{aligned}
$$$$
\times\left[1+\frac{1}{\tau} \int_{0}^{t} \exp \left\{\left(\frac{t}{\theta}\right)^{\beta}+\frac{t}{\tau}\right\} d t\right]
$$

Since historical data is imprecise and vague, so have some sort of uncertainties and consequently RAM parameters and their corresponding RAM-index also have some sorts of 


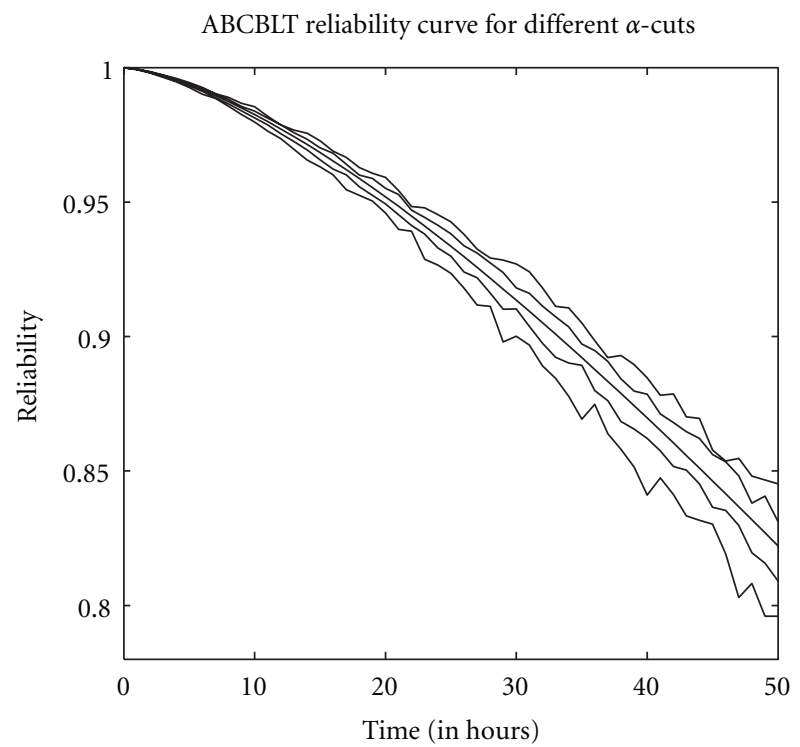

(a)

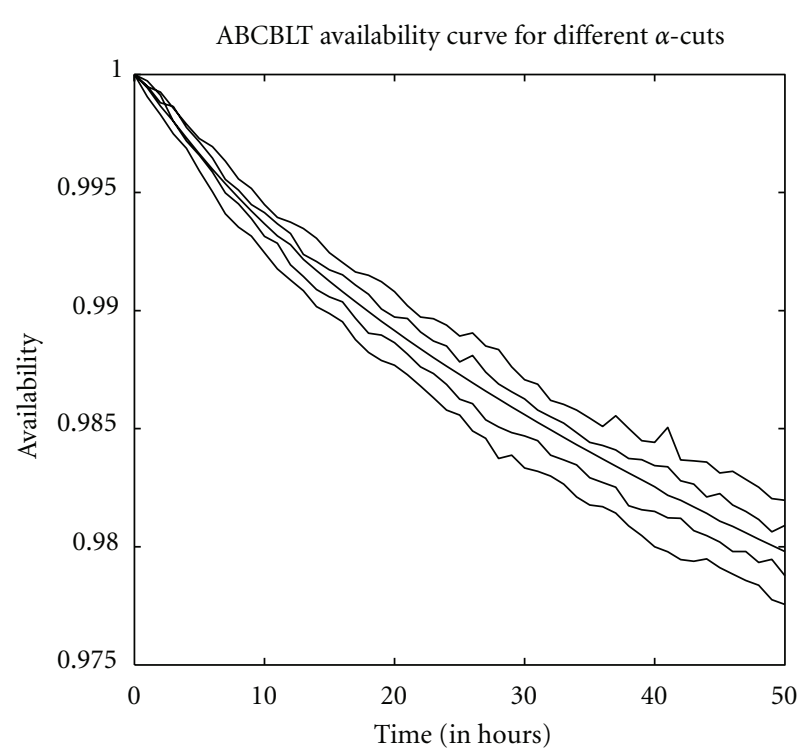

(b)

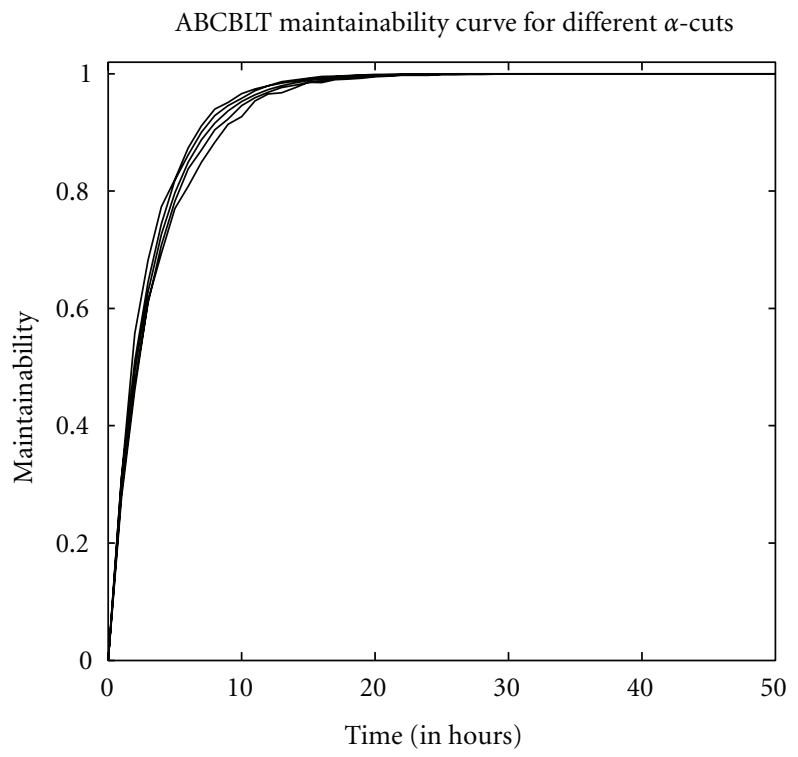

(c)

FIGURE 5: Long run period of RAM parameters at different $\alpha$ cuts.

uncertainties. For removing this, fuzzy set theory can be used to represent the uncertain data by taking crisp data into triangular fuzzy numbers. By using quantified data, RAM parameters and consequently RAM-Index becomes a triangular fuzzy membership function which can be expressed as

$$
\widetilde{\operatorname{RAM}}(t)=\left(\operatorname{RAM}_{L}(t), \operatorname{RAM}_{M}(t), \operatorname{RAM}_{R}(t)\right)
$$

It is clear from the (9) that at any time " $t$ ", $\operatorname{RAM}(t) \in$ $(0,1)$.

\section{ABCBLT Technique}

Lambda-Tau is a traditional methodology in which FTA is used to model the system. The basic expression used for analyzing the system based on constant failure rate model, that is, failure rate and repair time, follows the exponential distribution are summarized in Table 2. Knezevic and Odoom [11] extend this idea through PNs and fuzzy set theory for analyzing the various reliability parameters of the complex repairable industrial systems in the form of fuzzy membership functions. In their theory, PN is used for modeling the system while fuzzy takes care of impreciseness present in the data. They calculated the various reliability 
TABLE 3: Input data of the system.

\begin{tabular}{|c|c|c|c|}
\hline \multirow{3}{*}{ Components } & \multicolumn{2}{|c|}{ Failure data } & \multirow{3}{*}{$\begin{array}{c}\text { Repair time } \\
\text { MTTR } \\
\tau \text { (hrs) }\end{array}$} \\
\hline & \multicolumn{2}{|c|}{ Weibull parameters } & \\
\hline & scale $(\theta)$ & shape $(\beta)$ & \\
\hline Filter & 337 & 1.33 & 2.0 \\
\hline Screener & 315 & 1.54 & 4.0 \\
\hline Cleaner & 470 & 1.88 & 2.0 \\
\hline Deckers & 252 & 1.76 & 5.0 \\
\hline
\end{tabular}

TABLE 4: Decrease in spread for the parameters.

\begin{tabular}{lccc}
\hline \multirow{2}{*}{ Technique } & \multicolumn{3}{c}{ Computed spread for reliability indices } \\
& Reliability & Availability & Maintainability \\
\hline FLT & 0.00790434 & 0.00161782 & 0.04578209 \\
ABCBLT & 0.00361200 & 0.00069999 & 0.01876803 \\
\hline \multicolumn{3}{c}{ Decrease in spread in \% from FLT to ABCBLT } \\
\hline & 54.30358511 & 56.73251659 & 59.00573783 \\
\hline
\end{tabular}

parameters in the form of fuzzy membership functions which are used for behavior analysis of the repairable industrial system [2]. Their approach is limited as the number of components of the system increases or system structure becomes more complex, the computed reliability indices in the form of fuzzy membership function have wide spread $[14,16,17]$ due to various fuzzy arithmetic operations used in the calculations. Thus this approach is not suitable for the behavior analysis of large and complex repairable industrial systems when data is imprecisely known and represented by fuzzy numbers.

To generalize the approach for a complex industrial system, an effective technique is needed which should reduce the uncertainty level so that plant personnel may use these indices to analyze the system's behavior in more promising way for improving the system's performance. ABCBLT [17] is a hybridized technique in which LambdaTau methodology is used for obtaining the expression of various RAM parameters and artificial bee colony is used to solve the nonlinear programming problem for constructing their membership function by using ordinary arithmetic operations instead of fuzzy arithmetic operations. Triangular membership functions has been used for fuzzifying the data because it is easy to handle the information and form an accurate results in reliability engineering.

Strategy followed through this approach has been described through flowchart in Figure 2 and their details are given hereafter.

Step 1 (Information extraction phase). In the first phase of the system, the information related to failure rate and repair time of the system components are collected from the various resources such as logbooks, historical records, sheets, and so forth.

Step 2 (Fuzzifying the data). As the extracted data is either out of date or does not represent the actual failure of the system it leads the problem of uncertainty in the current failure rates and repair times. So, to handle the uncertainties in the analysis, the obtained collected (crisp) data is fuzzified into triangular fuzzy numbers (TFNs) having known spread (support) as suggested by decision maker/design maintenance expert/system reliability analyst in the form of the spread $( \pm 15 \%, \pm 25 \%$, and $\pm 50 \%)$.

Step 3 (Calculate RAM parameters). In this step, system is modelled with the help of Petri nets and based on that minimal cut sets are obtained by using matrix method. By using these cut sets and the expression of the LambdaTau methodology, RAM parameters of the system, listed in Table 1, are obtained. Instead of constructing the membership functions by using fuzzy arithmetic function, an ordinary arithmetic and optimization technique have been used for avoiding the high level of uncertainties existing in the computed reliability indices. For this a nonlinear programming problem (11) has been formulated by utilizing the quantified fuzzy $\theta^{\prime} s$ and $\tau^{\prime} s$. Thus, the lower and upper boundary values of reliability indices are computed at cut level $\alpha$ by solving

$$
\begin{aligned}
\text { minimize/maximize } & \widetilde{F}\left(\theta_{1}, \theta_{2}, \ldots, \theta_{n}, \tau_{1}, \tau_{2}, \ldots, \tau_{m}\right) \text { or } \\
& \widetilde{F}\left(t / \theta_{1}, \theta_{2}, \ldots, \theta_{n}, \tau_{1}, \tau_{2}, \ldots, \tau_{m}\right)
\end{aligned}
$$

subject to $\mu_{\theta_{i}}(x) \geq \alpha$,

$$
\begin{aligned}
& \mu_{\tau_{j}}(x) \geq \alpha, \\
& 0 \leq \alpha \leq 1, \\
& i=1,2, \ldots, n, \\
& j=1,2, \ldots, m,
\end{aligned}
$$

where $\widetilde{F}\left(\theta_{1}, \theta_{2}, \ldots, \theta_{n}, \tau_{1}, \tau_{2}, \ldots, \tau_{m}\right)$ and $\widetilde{F}\left(t / \theta_{1}, \theta_{2}, \ldots, \theta_{n}\right.$, $\left.\tau_{1}, \tau_{2}, \ldots, \tau_{m}\right)$ are time independent and dependent fuzzy reliability indices. The obtained minimum and maximum value of $\widetilde{F}$ are denoted by $F_{\min }$ and $F_{\max }$, respectively.

The membership function values of $\widetilde{F}$ at $F_{\max }$ and $F_{\min }$ are both $\alpha$, that is,

$$
\mu_{\widetilde{F}}\left(F_{\max }\right)=\mu_{\widetilde{F}}\left(F_{\min }\right)=\alpha .
$$

Since the problem is nonlinear in nature so it requires an efficient technique to solve this problem. Variety of methods and algorithms exists for optimization of such problems and applied in various technological fields. In this paper $A B C$ $[18-20,24]$ is used as a tool to find out the optimal solution of the above optimization problems, since $\mathrm{ABCs}$ have the advantages of memory, multi-character, local search, and solution improvement mechanism, it is able to discover an excellent optimal solution. The procedure of $\mathrm{ABC}$ algorithm is described in Algorithm 1. The objective function for maximization problem and the reciprocal of the objective function for minimization problem is taken as the fitness function. The termination criterion has been used either to a maximum number of generations or order of relative error equal to $10^{-6}$, whichever is achieved first. 
TABLE 5: Crisp and defuzzified values of RAM parameters at different spreads.

\begin{tabular}{|c|c|c|c|c|}
\hline \multirow{2}{*}{ Parameters } & \multirow{2}{*}{ Crisp values } & \multicolumn{3}{|c|}{ Defuzzified values at spread } \\
\hline & & $\pm 15 \%$ & $\pm 25 \%$ & $\pm 50 \%$ \\
\hline \multirow{2}{*}{ Reliability } & \multirow{2}{*}{0.98251623} & Lambda-Tau: 0.98215233 & 0.98145762 & 0.97708411 \\
\hline & & ABCBLT: 0.98249979 & 0.98214257 & 0.97947532 \\
\hline \multirow{2}{*}{ Availability } & \multirow{2}{*}{0.99367854} & Lambda-Tau: 0.99362948 & 0.99353884 & 0.99304228 \\
\hline & & ABCBLT: 0.99363929 & 0.99354976 & 0.99302078 \\
\hline \multirow{2}{*}{ Maintainability } & \multirow{2}{*}{0.95261602} & Lambda-Tau: 0.95147966 & 0.94946055 & 0.94010346 \\
\hline & & ABCBLT: 0.95184158 & 0.95117712 & 0.94583763 \\
\hline
\end{tabular}

TABle 6: Change in defuzzified values of parameters (in magnitude).

\begin{tabular}{lccc}
\hline \multicolumn{4}{c}{ Change in the reliability } \\
Parameters & I to II & I to III & II to III \\
\hline Reliability & 0.03703755 & 0.00167325 & 0.03537740 \\
Availability & 0.00493721 & 0.00394996 & 0.00098728 \\
Maintainability & 0.11928835 & 0.08129613 & 0.03803759 \\
\hline
\end{tabular}

I: crisp, II: Lambda-Tau III: ABCBLT.

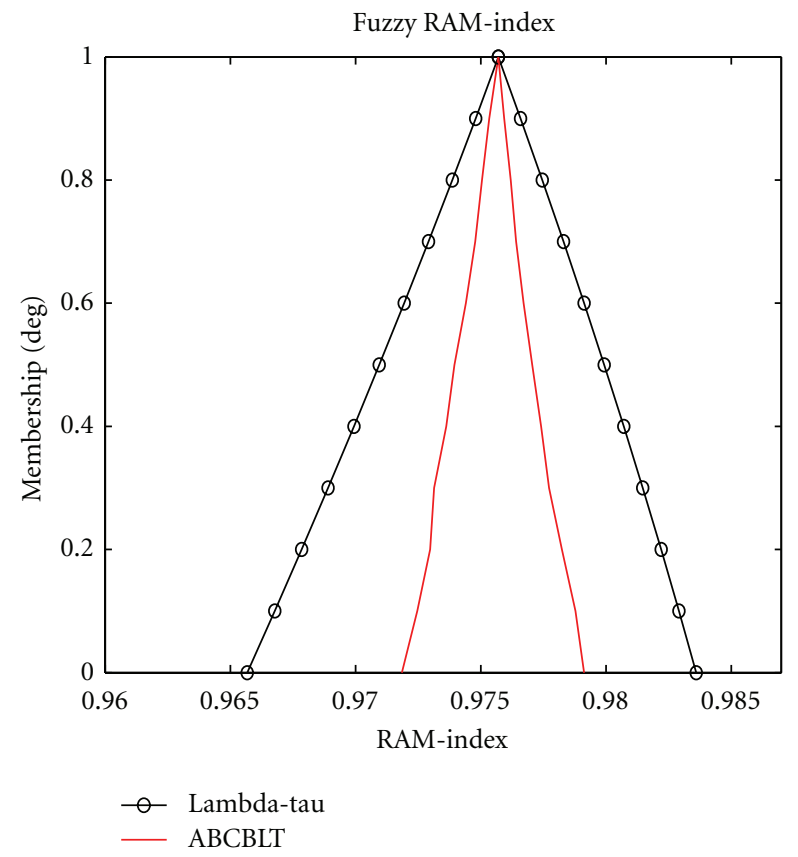

Figure 6: Fuzzy RAM-Index plot at $\pm 15 \%$ spread.

Step 4 (Defuzzification). As the collected output from the Step 3 of the methodology is of fuzzy output. But in real life, as most of the actions or decisions implemented by human or machines are binary or crisp it is necessary to convert the fuzzy output in crisp output. The process of converting the fuzzy output to crisp is called defuzzification. Out of existence of the several method for defuzzification, center of gravity (COG) method is selected due to its property that it is equivalent to mean of data and so it is very appropriate for reliability calculations [25]. If the membership function
$\mu_{\tilde{A}}(x)$ of the output fuzzy set $\tilde{A}$ is described on the interval $\left[x_{1}, x_{2}\right]$, then COG defuzzification $\bar{x}$ can be defined as

$$
\bar{x}=\frac{\int_{x_{1}}^{x_{2}} x \cdot \mu_{\widetilde{A}}(x) d x}{\int_{x_{1}}^{x_{2}} \mu_{\widetilde{A}}(x) d x} .
$$

\section{An Illustration with Application}

In the present study a paper plant situated in northern part of India producing 200 tons of paper per day is considered as the subject of discussion. The paper plants are large capital-oriented engineering system, comprising of subsystems, namely, chipping, feeding, pulping, washing, screening, bleaching, production of paper consisting of press unit, and collection, arranged in complex configuration. The present paper considers the most important functionary unit, namely, screening unit as a subject of discussion.

5.1. Screening System. The screening unit consists of four subsystems whose described are given as below:

(i) Filter (A): it works for removal of black liquor from the pulp. Its failure causes failure of the system.

(ii) Screen (B): it removes the knots and other undesirable materials from the pulp. Failure of the screen causes complete failure of the system.

(iii) Cleaner (C): it consists of three units in parallel. The failure of any one unit reduces the efficiency of the plant. Complete failure of the cleaner reduces the efficiency of the plant but the system remains operative. Manual operation is possible during the repair. Water is mixed here with the pulp by centrifugal action.

(iv) Decker (D): it reduces the blackness of the pulp. The failure of decker causes the complete failure of the system.

The reliability block diagram and its equivalent Petri net model are shown in Figures 3(a) and 3(b), respectively, where SSF represents the top place event of the screening unit system failure.

5.2. RAM Parameters Analysis. Under the information extraction phase, the data related to parameters of failure rate $\left(\beta_{i}, \theta_{i}\right)$ and repair time $\left(\tau_{i}\right)$ of the main component of the system are collected from the historical records and are 


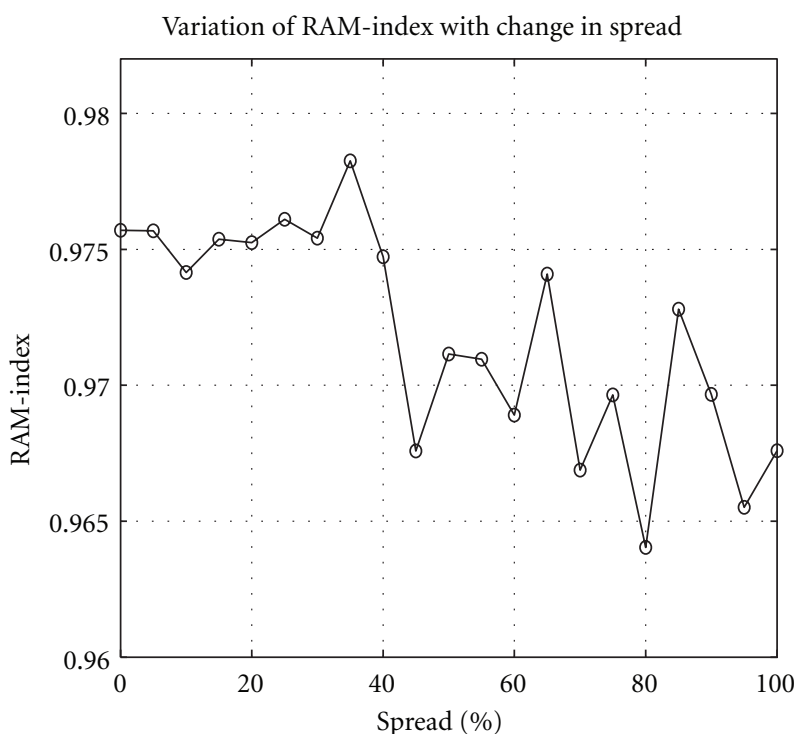

(a)

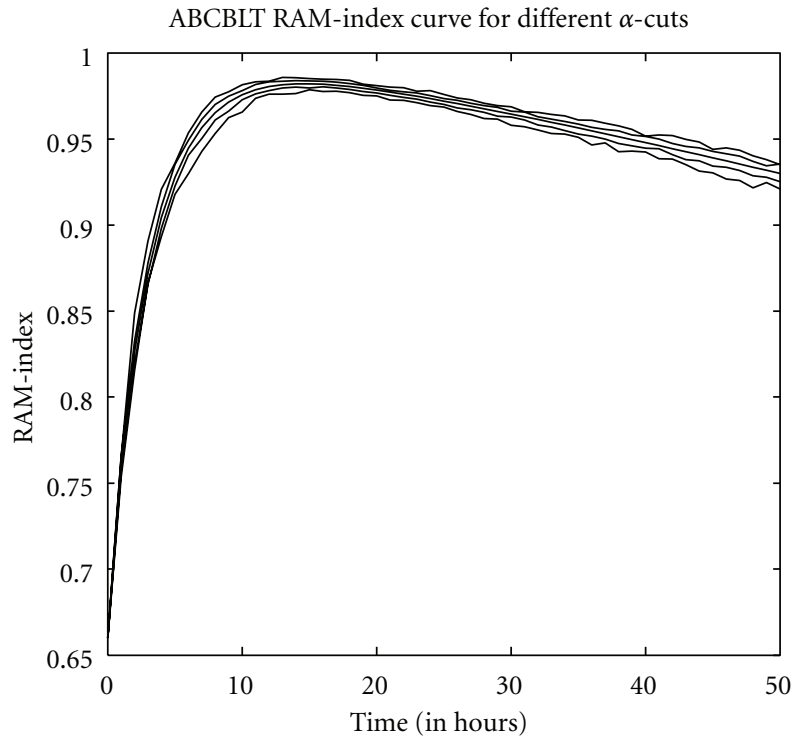

(b)

FIGURE 7: Variation of RAM-Index with (a) change in spread (b) different level of uncertainties.

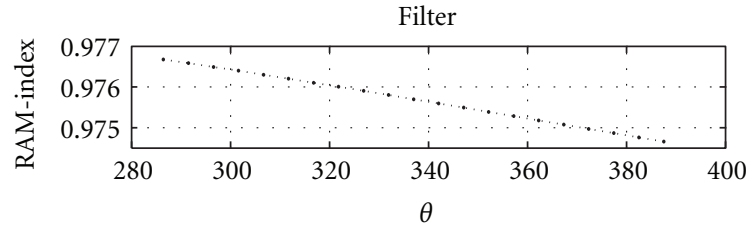

(a)

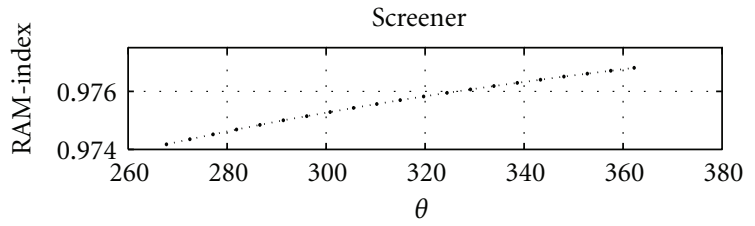

(c)

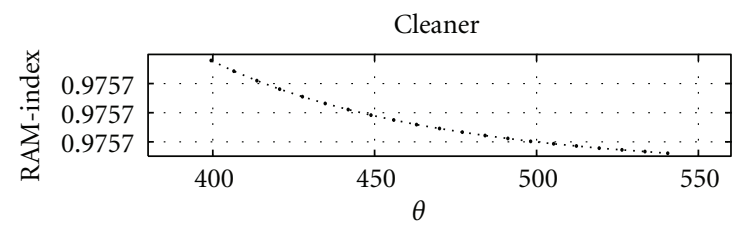

(e)

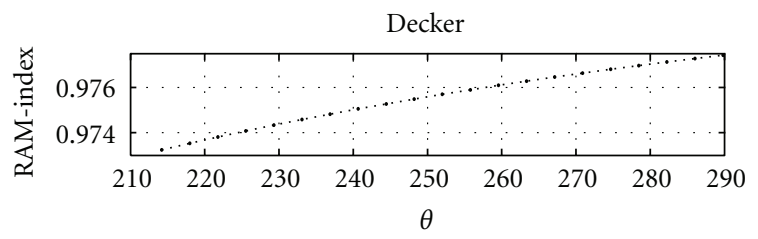

(g)

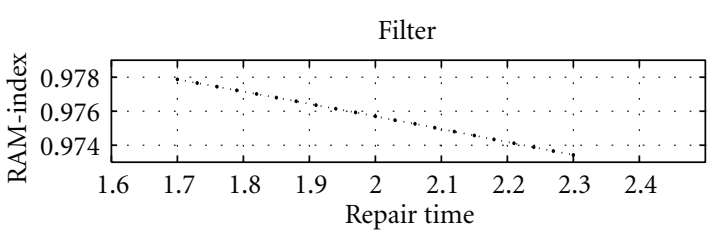

(b)

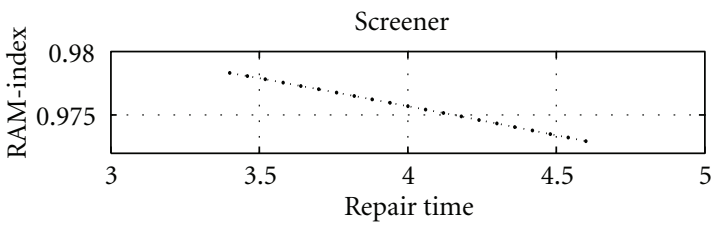

(d)

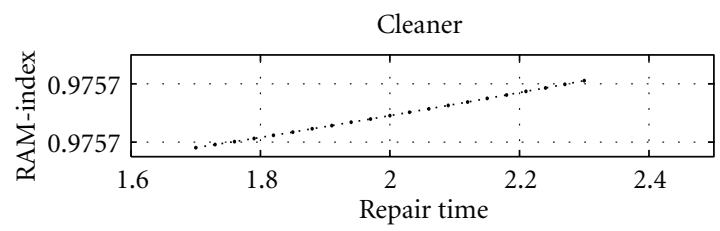

(f)

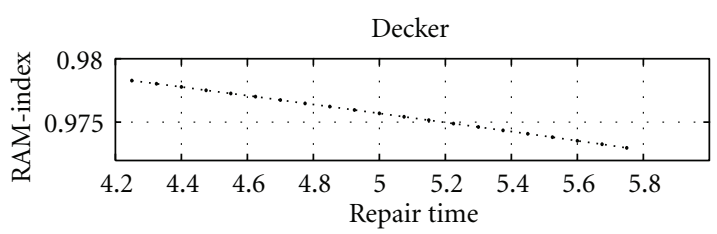

(h)

FIGURE 8: Effect of components parameters on RAM-Index when varies separately. 
TABLE 7: Range of RAM-Index when parameters of components varies separately.

\begin{tabular}{|c|c|c|c|c|}
\hline Component & Range of scale parameter $\theta$ (hrs) & RAM-Index & Range of repair time $\tau$ (hrs) & RAM-Index \\
\hline \multirow{2}{*}{ Filter } & \multirow{2}{*}{$286.45-387.55$} & Min: 0.97465822 & \multirow{2}{*}{$1.70-2.30$} & Min: 0.97342489 \\
\hline & & Max: 0.97667539 & & Max: 0.97787954 \\
\hline \multirow{2}{*}{ Screener } & \multirow{2}{*}{$267.75-362.25$} & Min: 0.97417942 & \multirow{2}{*}{$3.40-4.60$} & Min: 0.97294317 \\
\hline & & Max: 0.97680809 & & Max: 0.97831576 \\
\hline \multirow{2}{*}{ Cleaner } & \multirow{2}{*}{$399.50-540.50$} & Min: 0.97569885 & \multirow{2}{*}{$1.70-2.30$} & Min: 0.97569885 \\
\hline & & Max: 0.97569885 & & Max: 0.97569885 \\
\hline \multirow{2}{*}{ Decker } & \multirow{2}{*}{$214.20-289.80$} & Min: 0.97324561 & \multirow{2}{*}{$4.25-5.75$} & Min: 0.97298904 \\
\hline & & Max: 0.97743319 & & Max: 0.97827127 \\
\hline
\end{tabular}

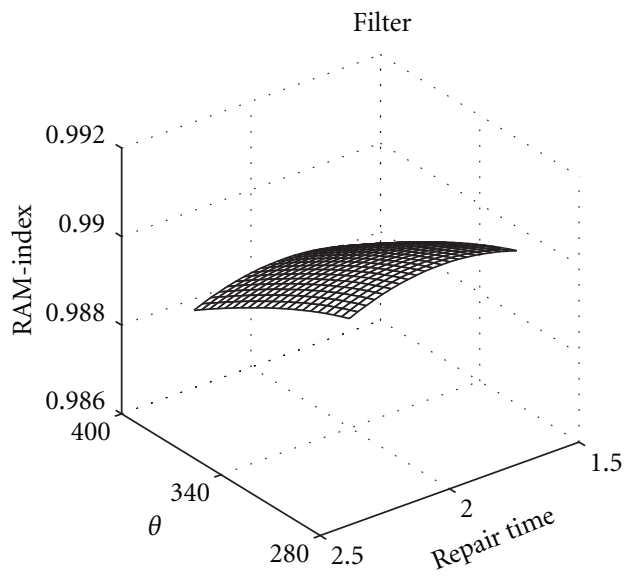

(a)

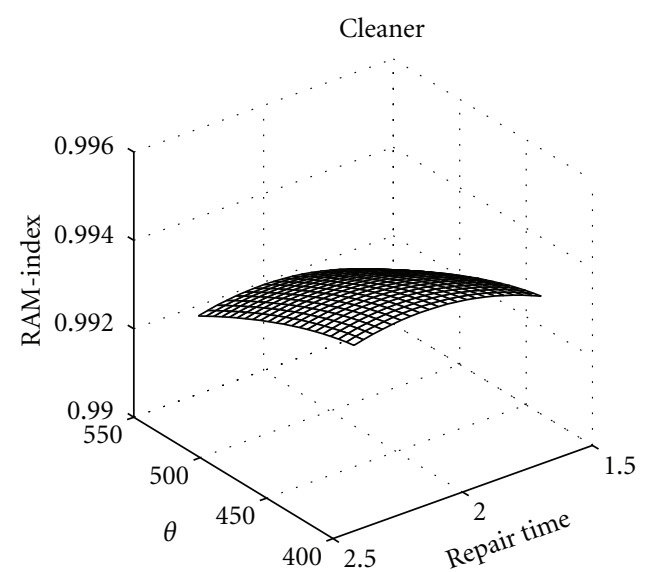

(c)

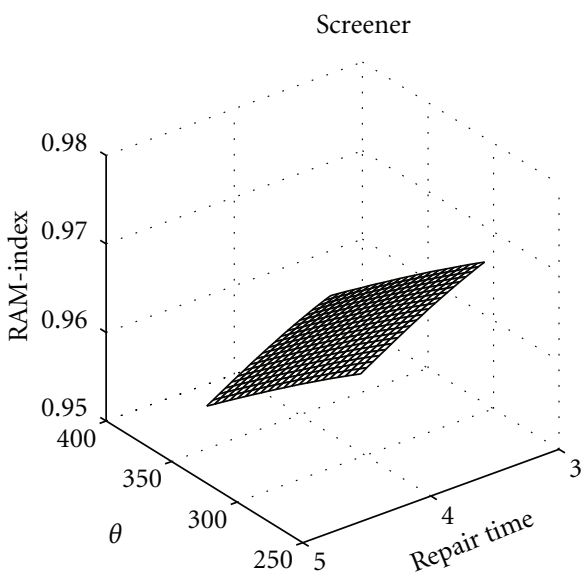

(b)

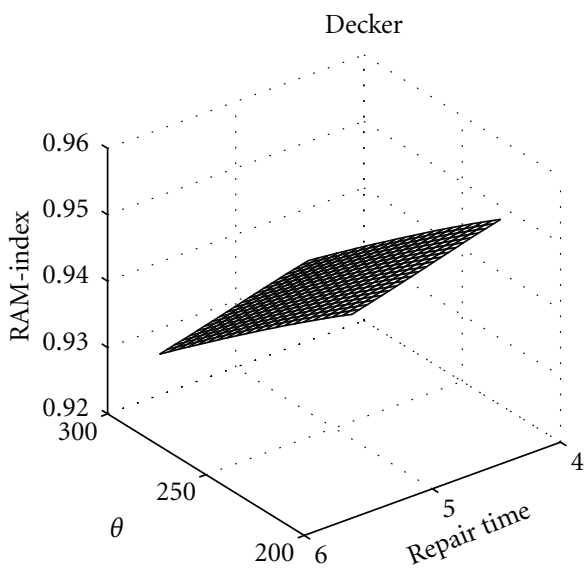

(d)

FIGURE 9: Effect of simultaneously varying components parameters on RAM-Index.

integrated with expertise of the system manager shown in Table 3.

As the collected data are taken from various resources which are out of data or contains large amount of uncertainty. So to handle these uncertainties and vagueness, the obtained (crisp) data are fuzzifier into fuzzy number with some known spread $\pm 15 \%$ as suggested by the decision maker or system analysts. The minimal cut sets of the system are obtained by using matrix method are $\{A\},\{B\}$, $\left\{C_{1} C_{2} C_{3}\right\}$, and $\{D\}$. By using these sets and following the basic steps of ABCBLT technique, RAM parameters of the system are obtained in the form of fuzzy membership functions, at various membership grades for the mission time $t=10$ (hrs) with left and right spread. The computed results by ABCBLT are depicted graphically in Figure 4 for $\pm 15 \%$ spreads along with Lambda-Tau results. From the 
TABLE 8: Range of RAM-Index when parameters of components varies simultaneously.

\begin{tabular}{|c|c|c|c|}
\hline Component & Range of scale parameter $\theta$ (hrs) & Range of repair time $\tau$ (hrs) & RAM-Index \\
\hline \multirow{2}{*}{ Filter } & \multirow{2}{*}{$286.45-387.55$} & \multirow{2}{*}{$1.70-2.30$} & Min: 0.98675020 \\
\hline & & & Max: 0.99197856 \\
\hline \multirow{2}{*}{ Screener } & \multirow{2}{*}{$267.75-362.25$} & \multirow{2}{*}{$3.40-4.60$} & Min: 0.95034946 \\
\hline & & & Max: 0.97417884 \\
\hline \multirow{2}{*}{ Cleaner } & \multirow{2}{*}{$399.50-540.50$} & \multirow{2}{*}{$1.70-2.30$} & Min: 0.99011339 \\
\hline & & & Max: 0.99565120 \\
\hline \multirow{2}{*}{ Decker } & \multirow{2}{*}{$214.20-289.80$} & \multirow{2}{*}{$4.25-5.75$} & Min: 0.92456143 \\
\hline & & & Max: 0.95706339 \\
\hline
\end{tabular}

figure it has been concluded that the computed results have reduce region and have a smaller spread than the LambdaTau results.

The decrease in spread of the RAM parameters by ABCBLT technique from the Lambda-Tau technique have been calculated and shown in tabulated form in Table 4. From the Table 4, it has been concluded that the largest and smallest decrease in spread occurs corresponding to the reliability and maintainability respectively. On the other hand, the largest and smallest spreads occurs corresponding to availability and maintainability for ABCBLT technique which means prediction range of reliability parameters decreased. This suggests that decision makers (DMs) have smaller and more sensitive region to make more sound and effective decision in lesser time and hence ABCBLT is a superior than Lambda-Tau technique.

For defuzzification, center of gravity method [25] is used because it has the advantage of being whole membership function into account for this transformation. The crisp and defuzzified values of RAM parameters at $\pm 15 \%, \pm 25 \%$, and $\pm 50 \%$ spreads are computed and compared with existing Lambda-Tau results which are shown in Table 5. It is clearly seen from the table that the result proposed by ABCBLT technique act as a bridge between Markovian process (crisp) and fuzzy Lambda-Tau (FLT) results. Also, variation in defuzzified values by ABCBLT technique are not so much as shown by results of Lambda-Tau technique, when the uncertainty level increases in the form of spread from $\pm 15 \%$ to $\pm 25 \%$, and further $\pm 50 \%$. In addition to this, Table 5 reflects that the crisp values do not change irrespective of the spread chosen while the defuzzified values change with change of spreads. The change in the value of RAM parameters of ABCBLT results from the crisp and Lambda-Tau results have been computed and presented in Table 6. Based on these results the system analyst/decision maker may change their target goals rather comes from the traditional analysis. For an example, if plant personnel want to optimize reliability of the system using ABCBLT results then the new target of system reliability should be greater than 0.98249979 rather 0.98215233 comes from Lambda-Tau when uncertainty level taken as $\pm 15 \%$ percent. Due to this and their reduced region of prediction, the value obtained through ABCBLT technique are conservative in nature which may be beneficial for system expert/analyst for future course of action that is, now the maintenance will be based on the defuzzified values rather than crisp values.

At different $\alpha$-cuts, $(0,0.5,1)$, reliability, availability and maintainability curves for $0-50$ (hrs) have been computed using ABCBLT technique and plotted in Figure 5 for depicting the behavior of the system with different levels of uncertainties. The behavior of these curves, using current conditions and uncertainties, shows that if current condition of equipments and subsystems are not changed then reliability of the systems will decrease rapidly while maintainability behave almost linearly after certain time for a long run period. The analysis suggests that to enhance the performance of these systems, current condition of equipments and subsystems should be changed according to effective maintenance program. But the problem is how to find the components or subsystems on which more attention should be given to save money, manpower and time for the effectiveness of the maintenance program. To overcome this problem a RAM analysis has been carried out using the proposed RAM-Index analysis.

5.3. RAM-Index Analysis. In order to analyze the effect of the system parameters on system performance, the RAMIndex analysis has been done. For this, the RAM-Index as given in (9) has been used. As the system analyst want to operated the system for a long run period for enhancing the production as well as productivity of the system. For this it is necessary that system should run failure free or have less range of uncertainties upto a desired levels. But failure is an unavoidable phenomenon, so in order to analyze the behavior of the system it is necessary that uncertainty levels in the analysis should be reduced upto a desired degree of accuracy. Hence fuzzy RAM-Index is computed by ABCBLT technique and their results are compared with Lambda-Tau results in Figure 6 at $\pm 15 \%$ spread. It has been concluded that the uncertainties level by the proposed technique has a reduced region than Lambda-Tau. Moreover, to see the behavior of RAM index against different uncertainty (spread) levels, a plot between spread from 0 to 100 (in \%) and RAM index has been plotted and shown in Figure $7(\mathrm{a})$. The variation of RAM-Index with a time range of $0-50$ (hrs) using ABCBLT technique for depicting the behavior of the system is shown in Figure 7(b) which shows that RAM index 
increases from 0 to 50 (hrs) and then decreases. At time $t=$ $0 \mathrm{hrs}$, the RAM-Index is 0.66 while it reaches to maximum in the range $0.978586-0.983811$ at time $t=16 \mathrm{hrs}$ and then decreases after that. This analysis shows that in order to improve the performance of the system, current conditions and equipment should be changed after time $t=16$ (hrs).

In order to find the components, as per preferential order, on which more attention to be given for increasing the performance of the system, a sensitivity analysis on the RAM-Index has been done by varying the corresponding components failure rate and repair time parameters and fixing the other components parameter at the same time. The results thus obtained are shown graphically in Figure 8 which contains four subplots and each subplots has two subplots corresponding to failure and repair rate parameters of their component. The maximum and minimum values of the index during analysis has been obtained and given in Table 7 . It has been observed that variation in failure and repair times of the filter, screener, and decker components significantly affect the RAM-Index of the system. For instance, an increase in scale parameter of filter components from $286.45 \mathrm{hrs}$ to $387.55 \mathrm{hrs}$ and the reduction in MTTR of the same from $2.30 \mathrm{hrs}$ to $1.70 \mathrm{hrs}$ reduce the system RAM-Index by approximately $0.529 \%$. The effect of cleaners on system RAM-Index is found insignificant, because these unit have standby systems.

But in the real life modeling, the failure rate and repair time parameters affect simultaneously the system performance. For this, an analysis has been done on RAMIndex by varying simultaneously the parameters of failure and repair rate. The effects of individual component of the system on the system performance is noticed and shown in graphically in Figure 9 while their ranges corresponding to their components are tabulated in Table 8. On the basis of results, it can be analyzed that for improving the performance of the screening system, more attention should be given to the components as per the preferential order; decker, screener, filter, and cleaner.

\section{Conclusion}

In the present study an investigation has been done on the RAM analysis of the screening unit in paper industry by utilizing uncertain, vague, and limited data. The uncertainties in the collected or available data are removed with the help of fuzzy numbers. The development of fuzzy numbers from the available data and using fuzzy possibility theory can greatly increase the relevance of reliability study. RAM parameters of the system have been calculated by using ABCBLT technique and results are compared with Lambda-Tau results. The major advantage of this technique is that it optimizes the spread of the reliability indices upto a desired degree of accuracy which indicates the higher sensitivity zone and thus may be useful for the reliability engineers/experts to make more sound decisions. Plant personnel may be able to predict the system behavior more precisely and will plan future maintenance. To enhance the system performance, critical components of the system as per the preferential order have been found by using proposed RAM-Index. Using RAMIndex, to improve the performance of the screening unit, more attention should be given in preferential order to the components; decker, screener, filter, and cleaner. Computed results will facilitate the management in reallocating the resources, making maintenance decisions, achieving long run availability of the system, and enhancing the overall productivity of the paper mill. These results will also help the concerned plant managers to plan and adapt suitable maintenance strategies for improving system performance and thereby reduce operational and maintenance costs.

\section{References}

[1] D. Kumar, Analysis and optimization of systems availability in sugar, paper and fertilizer industries [Ph.D. thesis], University of Roorkee (Presently IIT Roorkee), Roorkee, India, 1991.

[2] C. Ebeling, An Introduction to Reliability and Maintainability Engineering, Tata McGraw-Hill, New York, NY, USA, 2001.

[3] H. Garg and S. P. Sharma, "RAM analysis of a coal crushing unit of a thermal power plant using fuzzy Lambda-Tau Methodology," in Proceedings of the 1st International Conference on Emerging Trends in Mechanical Engineering (ICETME '11), pp. 795-802, Thapar University, Patiala, India.

[4] H. Garg and S. P. Sharma, "A two-phase approach for reliability and maintainability analysis of an industrial system," International Journal of Reliability, Quality and Safety Engineering, vol. 19, no. 3, 2012.

[5] A. Adamyan and D. He, "Analysis of sequential failures for assessment of reliability and safety of manufacturing systems," Reliability Engineering and System Safety, vol. 76, no. 3, pp. 227-236, 2002.

[6] A. Adamyan and D. He, "System failure analysis through counters of Petri net models," Quality and Reliability Engineering International, vol. 20, no. 4, pp. 317-335, 2004.

[7] H. Garg, "Reliability analysis of repairable systems using Petri nets and Vague Lambda-Tau methodology," ISA Transactions, http://dx.doi.org/10.1016/j.isatra.2012.06.009 . In press.

[8] P. S. Rajpal, K. S. Shishodia, and G. S. Sekhon, "An artificial neural network for modeling reliability, availability and maintainability of a repairable system," Reliability Engineering and System Safety, vol. 91, no. 7, pp. 809-819, 2006.

[9] Komal, S. P. Sharma, and D. Kumar, "RAM analysis of repairable industrial systems utilizing uncertain data," Applied Soft Computing Journal, vol. 10, no. 4, pp. 1208-1221, 2010.

[10] M. Rani, S. P. Sharma, and H. Garg, "Reliability analysis of press unit in paper plant using weibull fuzzy distribution function," in Proceedings of the 16th Online World Conference on Soft computing in Industrial Application (WSC '11), December 2011, http://wsc16.cs.lboro.ac.uk/conference/sites/ default/files/Paper_0.pdf.

[11] J. Knezevic and E. R. Odoom, "Reliability modelling of repairable systems using Petri nets and fuzzy Lambda-Tau methodology," Reliability Engineering and System Safety, vol. 73, no. 1, pp. 1-17, 2001.

[12] S. P. Sharma and H. Garg, "Behavioural analysis of urea decomposition system in a fertiliser plant," International Journal of Industrial and Systems Engineering, vol. 8, no. 3, pp. 271-297, 2011.

[13] H. Garg and S. P. Sharma, "Behavior analysis of synthesis unit in fertilizer plant," International Journal of Quality and Reliability Management, vol. 29, no. 2, pp. 217-232, 2012. 
[14] D. L. Mon and C. H. Cheng, "Fuzzy system reliability analysis for components with different membership functions," Fuzzy Sets and Systems, vol. 64, no. 2, pp. 145-157, 1994.

[15] H. Garg and S. P. Sharma, "Stochastic behavior analysis of complex repairable industrial systems utilizing uncertain data," ISA Transactions, vol. 51, no. 6, pp. 752-762, 2012.

[16] S. M. Chen, "Fuzzy system reliability analysis using fuzzy number arithmetic operations," Fuzzy Sets and Systems, vol. 64, no. 1, pp. 31-38, 1994.

[17] H. Garg, S. P. Sharma, and M. Rani, "Behavior analysis of pulping unit in a paper mill with weibull fuzzy distribution function using ABCBLT technique," International Journal of Applied Mathematics and Mechanics, vol. 8, no. 4, pp. 86-96, 2012.

[18] D. Karaboga, "An idea based on honey bee swarm for numerical optimization," Tech. Rep. TR06, Computer Engineering Department, Engineering Faculty, Erciyes University, 2005.

[19] D. Karaboga and B. Basturk, "A powerful and efficient algorithm for numerical function optimization: artificial bee colony (ABC) algorithm," Journal of Global Optimization, vol. 39, no. 3, pp. 459-471, 2007.

[20] D. Karaboga and B. Basturk, "On the performance of artificial bee colony (ABC) algorithm," Applied Soft Computing Journal, vol. 8, no. 1, pp. 687-697, 2008.

[21] L. A. Zadeh, "Fuzzy sets," Information and Control, vol. 8, no. 3, pp. 338-353, 1965.

[22] W. Pedrycz, "Why triangular membership functions?" Fuzzy Sets and Systems, vol. 64, no. 1, pp. 21-30, 1994.

[23] X. Bai and S. Asgarpoor, "Fuzzy-based approaches to substation reliability evaluation," Electric Power Systems Research, vol. 69, no. 2-3, pp. 197-204, 2004.

[24] D. Karaboga and B. Akay, "A comparative study of Artificial Bee Colony algorithm," Applied Mathematics and Computation, vol. 214, no. 1, pp. 108-132, 2009.

[25] T. J. Ross, Fuzzy Logic with Engineering Applications, John Wiley \& Sons, New York, NY, USA, 2nd edition, 2004. 

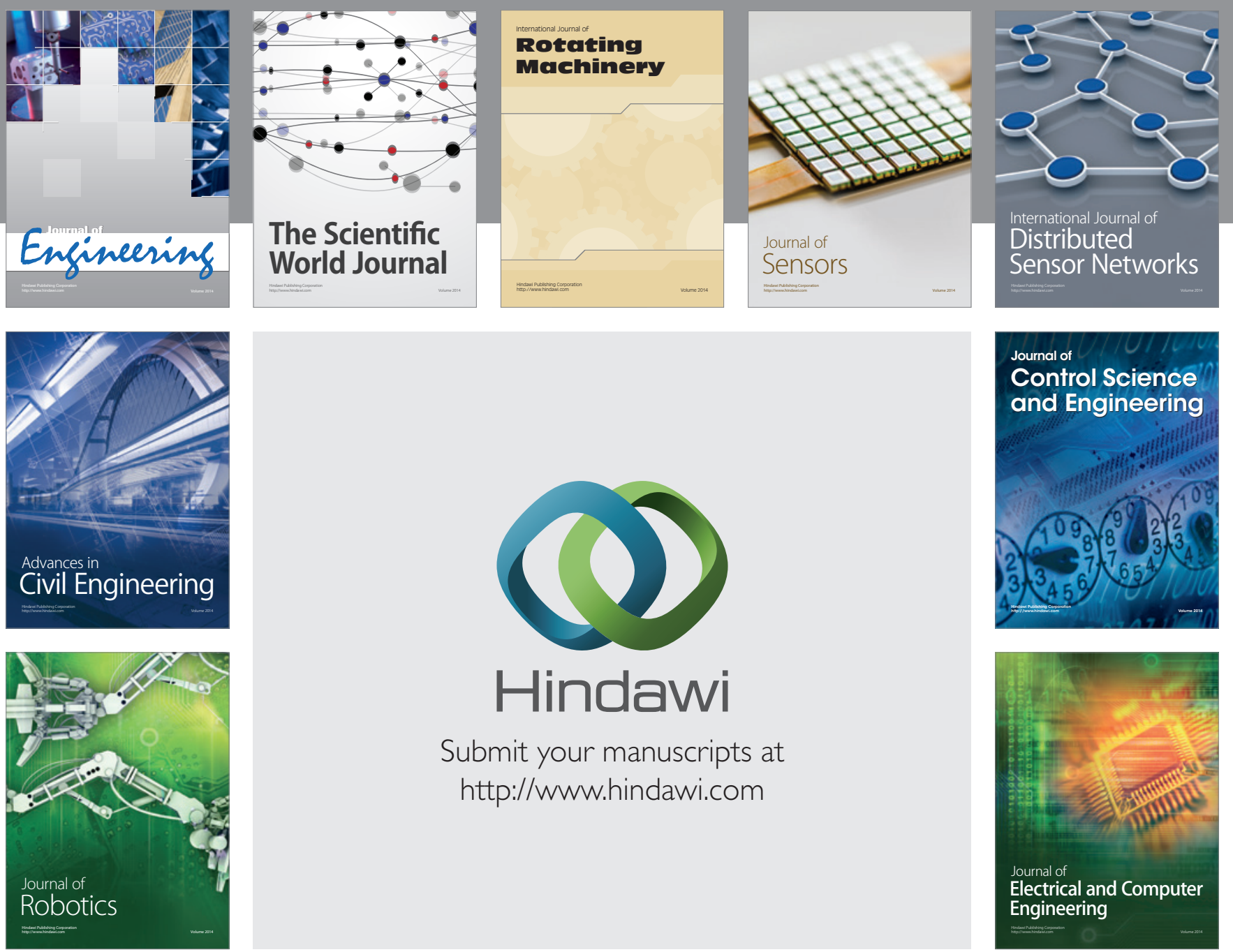

Submit your manuscripts at

http://www.hindawi.com
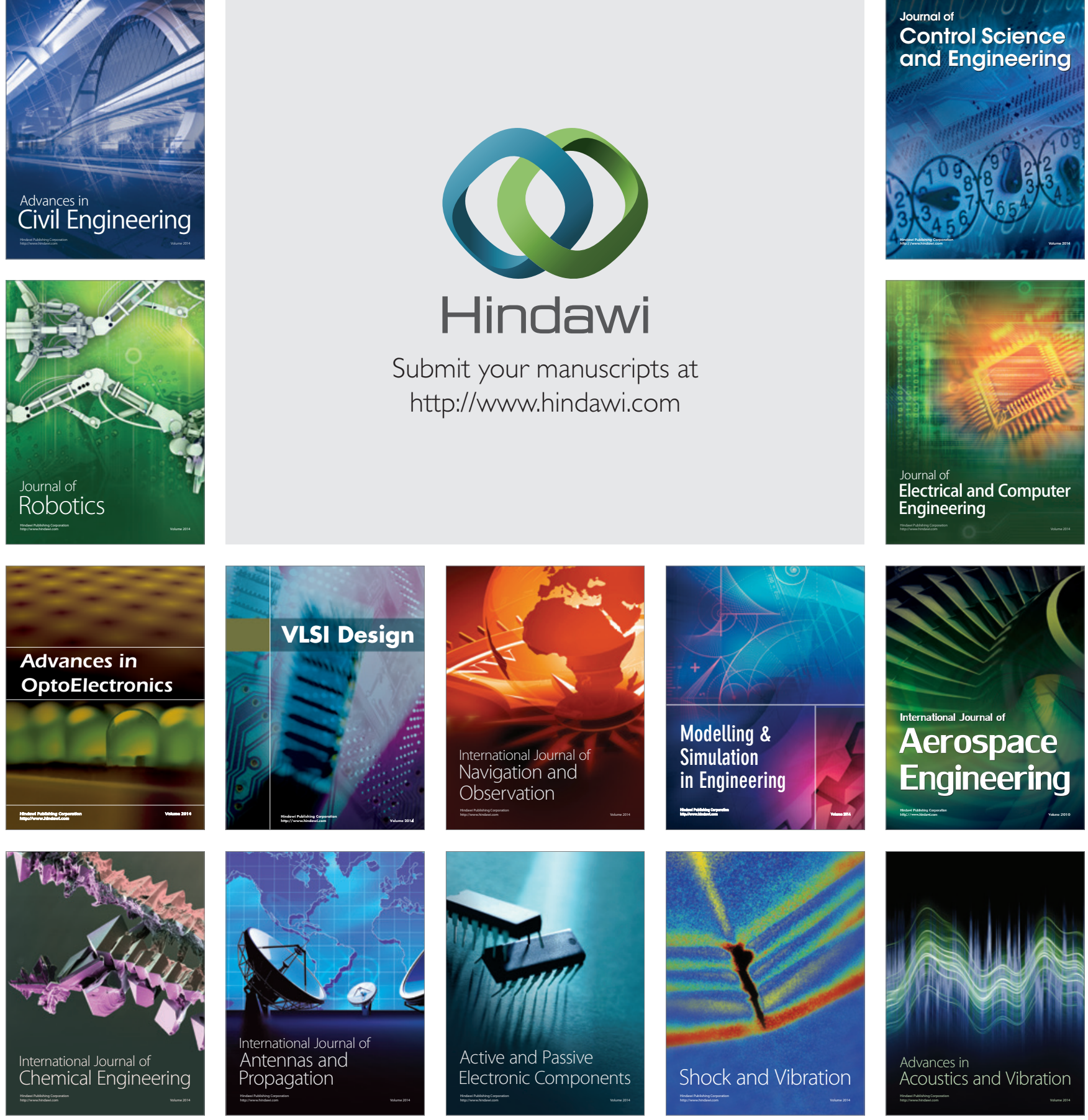